\section{Konsensuspapiere}

Monatsschr Kinderheilkd 2019 · 167:916-924 https://doi.org/10.1007/s00112-019-0749-6 Online publiziert: 5 . August 2019

๑) Springer Medizin Verlag GmbH, ein Teil von Springer Nature 2019

\section{Redaktion}

A. Borkhardt, Düsseldorf

S. Wirth, Wuppertal

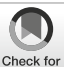

\author{
Harald Bertram ${ }^{1} \cdot$ Harald Sauer $^{2} \cdot$ Nikolaus Haas ${ }^{3,4}$ \\ ' Klinik für Pädiatrische Kardiologie und Pädiatrische Intensivmedizin, Medizinische Hochschule Hannover, \\ Hannover, Deutschland \\ ${ }^{2}$ Klinik für Pädiatrische Kardiologie, Kliniken für Kinder- und Jugendmedizin, Universitätsklinikum des \\ Saarlandes, Homburg/Saar, Deutschland \\ ${ }^{3}$ Abteilung Kinderkardiologie und Pädiatrische Intensivmedizin, Klinikum der Ludwig-Maximilians- \\ Universität, Campus Großhadern, München, Deutschland \\ ${ }^{4}$ Abteilung Kinderkardiologie und Pädiatrische Intensivmedizin, Klinikum der Ludwig-Maximilians- \\ Universität, Campus Innenstadt, München, Deutschland
}

\title{
Empfehlungen und Standards für die Analgosedierung kinderkardiologischer Patienten
}

\section{Positionspapier der Deutschen Gesellschaft für Pädiatrische Kardiologie und Angeborene Herzfehler (DGPK)}

\section{Kernaussage 1}

Kinderkardiologische Patienten tolerieren diagnostische und therapeutische Eingriffe in Analgosedierung oft besser als in Allgemeinanästhesie mit Intubationsnarkose

Da kinderkardiologische Patienten abhängig von der zugrunde liegenden Diagnose, möglichen Voreingriffen, ihrem aktuellen klinischen Zustand und des geplanten Eingriffs besondere Risikofaktoren für Prozeduren, die mit einer Sedierung einhergehen, aufweisen können, müssen diese bei Planung und Durchführung eines diagnostischen oder therapeutischen Eingriffs in Sedierung berücksichtigt werden.

Das primäre Zwischenfallrisiko gründet zum einen auf dem Nichterkennen von Risikofaktoren des kinderkardiologischen Patienten und zum anderen sekundär in einer zu tiefen Analgosedierung. Da eine vitale Gefährdung des Patienten aber jederzeit auftreten kann, müssen in jedem Fall ausreichende Vorkehrungen zu Monitoring des Patienten, Erkennung einer kardiopulmonalen Beeinträchtigung oder einer zu tiefen Sedierung des Patienten sowie zur erfolgrei- chen Behebung jeglicher Komplikation getroffen werden.

Obwohl eine gut titrierbare Analgosedierung mit z.B. Propofol als Sedativum und einem zusätzlichen Analgetikum (z. B. Ketamin) in der Mehrzahl der kardiologischen Zentren bereits seit Jahren klinischer Standard [3] und in den kinderkardiologischen Zentren seit Jahren gelebte Praxis ist [2,4-6], gibt es noch keine Empfehlungen oder Leitlinien über die Durchführung von Analgosedierungen im Rahmen von kinderkardiologischen Eingriffen seitens der zuständigen Fachgesellschaft.

Die Deutsche Gesellschaft für Pädiatrische Kardiologie und Angeborene Herzfehler (DGPK) legt daher in Ermangelung systematischer Studien zu diesem Thema mit diesem Positionspapier Empfehlungen und Standards zur Durchführung einer Analgosedierung bei kinderkardiologischen Patienten vor. Diese sollen grundsätzlich für alle Einrichtungen gelten, die derartige Eingriffe durchführen. Die Empfehlungen dieses Positionspapiers berücksichtigen eine aktuelle Umfrage unter den kinderkardiologischen Zentren und Kliniken Deutschlands. Hierbei wurden die gängige Sedierungspraxis, die verwen- 
Tab. 1 Auswahl diagnostischer oder therapeutischer Eingriffe, die einer Analgosedierung bedürfen können

Transthorakale Echokardiographie bei unruhigem Kind

Transösophageale Echokardiographie

Kardioversion

Pleurapunktion/-drainage

Perikardpunktion/-drainage

Diagnostischer/therapeutischer Herzkatheter

Testung von Defibrillator

CT-/MRT-Untersuchung

Implantation eines „event recorder"

Bronchoskopie

Anlage eines zentralen Venenkatheters

Verbandwechsel

Anlage einer periphervenösen Verweilkanüle

CT Computertomographie, MRT Magnetresonanztomographie

deten Pharmaka sowie die personellen, strukturellen und inhaltlichen Rahmenbedingungen erfragt [2].

\section{Sedierungsstufen}

Die Klassifikation der American Society of Anesthesiologists (ASA, [7]) ist Grundlage bestehender Leitlinien zur Einstufung von Sedierungsgraden bei erwachsenen und pädiatrischen Patienten. Die Sedierungstiefe entspricht einem Kontinuum von der minimalen bzw. moderaten Sedierung bei noch erweckbarem Patienten (Stufen I und II) über die tiefe Sedierung mit Bewusstseinsverlust des Patienten (Stufe III) und eingeschränkten Schutzreflexen bis zum Übergang zur Allgemeinanästhesie (Stufe IV) mit eingeschränkter Spontanatmung.

Die Analgosedierung kinderkardiologischer Patienten außerhalb von Kliniken für Kinder- und Jugendmedizin und Kinderherzzentren beschränkt sich in der Mehrzahl der Fälle auf die geplante leichte bis moderate Sedierungstiefe (Stufen I und II, z. B. für die Anlage einer periphervenösen Verweilkanüle [pVK] oder für eine Echokardiographie beim unruhigen und sich wehrenden Patienten).

Patienten ab der ASA-Sedierung-Stufe III haben ein deutlich erhöhtes Risiko- potenzial für Komplikationen während einer Sedierung bzw. Intervention. Im Vergleich zwischen tiefer Sedierung und Allgemeinanästhesie mit Intubation und der dafür notwendigen Medikation kann gerade bei kinderkardiologischen Patienten ein höheres Risiko für das letztgenannte Verfahren bestehen, weil die kardiologische Grunderkrankung bzw. die aktuelle klinische Situation mit einem erhöhten Risiko für eine hämodynamische Instabilität bei Allgemeinanästhesie einhergeht. Bei diesen Patienten bedingt eine Analgosedierung eine entsprechende Risikoreduktion für diagnostische oder therapeutische Eingriffe.

Analog zu den Empfehlungen verschiedener Fachgesellschaften empfehlen die Autoren eine verkürzte Einteilung der verschiedenen Sedierungsstadien gemäß der ASA (•Tab. 2):

Ein generelles Problem in der Zuordnung von erforderlichen Sedierungsstufen zu geplanten Prozeduren liegt in dem altersabhängig unterschiedlichen Bedarf an Analgosedierung im Kindes- und Jugendalter. Während ein Jugendlicher für einfache Eingriffe oft ähnlich wie ein Erwachsener behandelt werden kann und allenfalls eine geringe Analgosedierung oder ausschließlich eine adäquate Lokalanästhesie benötigt, erfordert die gleiche Maßnahme bei einem Kleinkind eine tiefe Sedierung mit entsprechend höherem Überwachungsbedarf.

Die individuelle Toleranz der Prozedur ist als Sedierungsziel daher in der Praxis wichtiger als eine Sedierungstiefe, die einer bestimmten Prozedur zugeordnet wird.

\section{Risikobeurteilung}

Die Risiken eines Eingriffs in Analgosedierung sind vom Sedierungsgrad, dem Alter des Patienten, der Eingriffsdauer und zusätzlich von der kardialen Grunderkrankung des Patienten abhängig, bei therapeutischen Prozeduren auch maßgeblich von der durchgeführten Intervention. [9]

Das Behandlungsteam muss fähig sein, prozedurale Komplikationen wie z. B. eine drohende kardiopulmonale Dekompensation des Patienten ebenso zu erkennen und adäquat zu behandeln wie
Folgen einer zu tiefen Sedierung. Hierzu gehören kardiozirkulatorisch z. B. Bradyoder Tachykardie und Hypotonie oder respiratorisch Brady- und Apnoe mit Hyperkapnie und Hypoxie. Zusätzlich müssen verfahrenstypische Komplikationen, wie z.B. Perikarderguss, Rhythmusstörungen etc., rechtzeitig erkannt und behandelt werden.

\section{Kernaussage 2}

Das individuelle Risiko einer diagnostischen oder therapeutischen Prozedur in Analgosedierung wird neben der Sedierungstiefe sowie der Art und Dauer des Eingriffs v. a. durch die zugrunde liegende Herzerkrankung, den aktuellen klinischen Status und das Alter des Patienten bestimmt.

Das Erkennen von Risikofaktoren, die einen Zwischenfall während der Analgosedierung begünstigen, ist deshalb von höchster Wichtigkeit. Eine vorausgehende Risikobeurteilung des Patienten mit Aktenstudium, Anamnese und einer gezielten körperlichen Untersuchung muss durchgeführt und dokumentiert werden. Die ärztliche Vorbereitung zur Analgosedierung umfasst bei allen Patienten:

- die detaillierte Anamneseerhebung

(u. a. Vitalfunktionen, Belastbarkeit, Infektionsstatus, Allergien, frühere Anästhesien/Sedierungen, aktuelle Medikationen),

- die Beurteilung des kardiozirkulatorischen Status (klinische Untersuchung, Elektrokardiographie [EKG], Echokardiographie, Blutdruck, ggf. weitere Befunde),

- die körperliche Untersuchung (insbesondere Beurteilung möglicher Herzinsuffizienzzeichen, Auskultation des Herzens und der Lungen, Inspektion des Mund-Rachen-Raums bezüglich Infektion und möglicher Probleme bei einer Atemwegssicherung, Venenverhältnisse),

- die adäquate Aufklärung (eine schriftliche Dokumentation im Rahmen der Aufklärung des Eingriffs ist ausreichend, jedoch müssen nachweislich die spezifischen Risiken einer tiefen Analgosedierung angesprochen worden sein). 
Monatsschr Kinderheilkd 2019 • 167:916-924 https://doi.org/10.1007/s00112-019-0749-6

(c) Springer Medizin Verlag GmbH, ein Teil von Springer Nature 2019

\section{H. Bertram · H. Sauer $\cdot$ N. Haas}

\section{Empfehlungen und Standards für die Analgosedierung kinderkardiologischer Patienten. Positionspapier der Deutschen Gesellschaft für Pädiatrische Kardiologie und Angeborene Herzfehler (DGPK)}

\section{Zusammenfassung}

Hintergrund. Bei kinderkardiologischen Patienten bzw. Kindern mit angeborenen Herzfehlern werden häufig diagnostische oder therapeutische Prozeduren durchgeführt, die einer Sedierung bzw. Analgesie bedürfen. Gerade diese Patientengruppe toleriert eine Analgosedierung häufig besser als eine Allgemeinanästhesie in Intubationsnarkose.

Ziel der Arbeit. Die Deutsche Gesellschaft für Pädiatrische Kardiologie und Angeborene Herzfehler (DGPK) erstellt Standards und Empfehlungen zur Durchführung der Analgosedierung kinderkardiologischer Patienten. Diese sollen grundsätzlich für alle Einrichtungen gelten, die derartige Eingriffe durchführen

Material und Methoden. Da systematische Studien fehlen, werden, basierend auf einer deutschlandweiten Umfrage zur gelebten
Praxis sowie nach Analyse der vorliegenden Literatur, die gängige Sedierungspraxis der üblichen Eingriffe, die verwendeten Pharmaka sowie die personellen, strukturellen und inhaltlichen Rahmenbedingungen analysiert und daraus Standards und Empfehlungen erarbeitet.

Ergebnisse. Das individuelle Risiko einer diagnostischen oder therapeutischen Prozedur in Analgosedierung wird neben der Sedierungstiefe sowie der Art und Dauer des Eingriffs v. a. durch die zugrunde liegende Herzerkrankung, den aktuellen klinischen Status und das Alter des Patienten bestimmt. Daraus resultiert das Ausmaß des periprozeduralen Monitorings. Empfehlungen zu Prozessqualität und postinterventioneller Überwachung werden definiert. Bei kompensierter kardiopulmonaler Situation, risikoarmen und/oder kurzen Prozeduren in moderater Sedierungstiefe besteht v. a. für Kinder jenseits der Säuglingsperiode kein erhöhtes Risikoprofil. Eine Analgosedierung kann unter ärztlicher Verantwortung an geschultes nichtärztliches Personal delegiert werden; abteilungsinterne Ausbildungscurricula mit interner Qualitätskontrolle werden empfohlen.

Schlussfolgerung. Eine Analgosedierung kinderkardiologischer Patienten ist bei der Mehrzahl der Eingriffe Methode der Wahl.

Schlüsselwörter

Risikobewertung · Diagnostische Techniken und Prozeduren · Interventionelle Prozeduren . Kardiales Risiko · Perioperative Versorgung

\section{Recommendations and standards for analgosedation of pediatric cardiac patients. Position Paper of the German Society for Pediatric Cardiology and Congenital Heart Disease (DGPK)}

\section{Abstract}

Background. In pediatric cardiology patients and patients with congenital heart defects, diagnostic procedures and therapeutic interventions are frequently performed. These measures often require adequate sedation and/or analgesia. It is well known that these children tolerate analgosedation better than general anesthesia with intubation.

Objective. The German Society for Pediatric Cardiology and Congenital Heart Disease (DGPK) establishes recommendations and defines standards for the periprocedural management of analgosedation in pediatric cardiology patients. These recommendations should serve as a standard for all institutions performing such procedures.

Material and methods. Due to a lack of systematic prospective studies the current best practice in Germany was analyzed based on a nationwide survey on this topic. The study analyzed the mode of sedation and analgesia, the relevant diagnostic and therapeutic procedures, the medication used, the monitoring, the personal and structural equipment and the general setting. In addition, the current literature was searched and standards and recommendations were defined based on the results.

Results. The individual risk of a diagnostic or interventional procedure under analgosedation is characterized by the intensity of sedation, the type and duration of the procedure, the underlying cardiac problem and the age and current clinical status of the patient. These factors define the extent of periprocedural monitoring. Recommendations for periprocedural management and postprocedural monitoring were elaborated. In patients with a compensated cardiopulmonary situation, for low and moderate risk procedures of short duration and moderate sedation, there is no additional risk profile especially in children more than 1 year old. Analgosedation can be delegated to and safely performed by trained nonmedical personnel under the responsibility of a physician. Dedicated standardized operational protocols (SOP) with quality assurance measures are strongly recommended.

Conclusion. Analgosedation is the method of choice for the majority of procedures in pediatric cardiology patients.

\section{Keywords}

Risk assessment - Diagnostic techniques and procedures · Interventions - Cardiac risk Perioperative care
Bei geplanten tiefen Sedierungen soll wegen des möglichen fließenden und unvorhersehbaren Übergangs zur Allgemeinanästhesie eine Vorbereitung erfolgen, die inhaltlich der einer Allgemeinanästhesie entspricht.

\section{Risikofaktoren}

Das Vorliegen von Risikofaktoren bedingt nicht automatisch die Notwendigkeit der Durchführung einer Allgemeinanästhesie mit Intubation; eine Allgemeinanästhesie kann gerade bei Patien- ten mit primär kardialen Risikofaktoren deren hämodynamische Situation verschlechtern. Kinderkardiologische Patienten mit kompensierter kardiopulmonaler Situation weisen per se für risikoarme und/oder kurze Prozeduren kein erhöhtes Risikoprofil auf. Für eine An- 


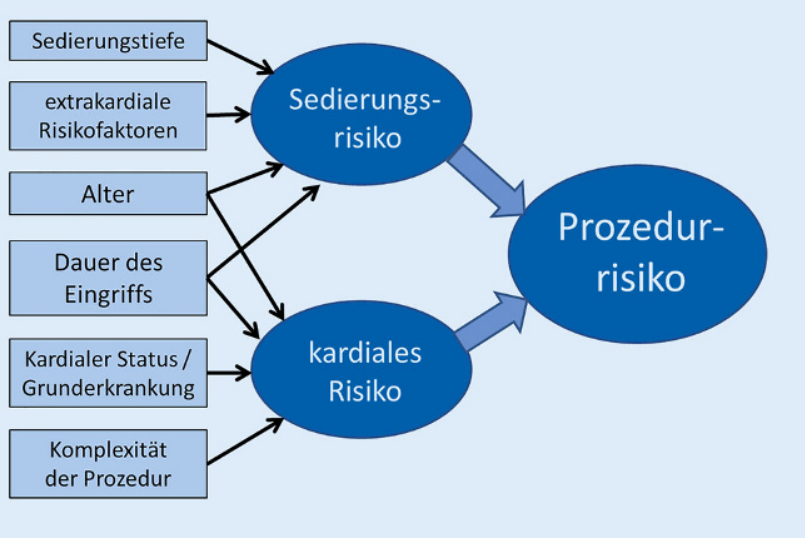

\section{Strukturqualität}

\section{Räumlichkeit}

Abb. $1 \triangleleft$ Risiken eines in Analgosedierung durchgeführten Eingriffs. Der Einfluss der einzelnen Risikofaktoren kann individuell stark variieren algosedierung müssen zusätzlich folgende Risikofaktoren in Abhängigkeit von der geplanten Intervention und Methode der Analgosedierung einbezogen sowie bei der Planung und Durchführung des Eingriffs berücksichtigt werden:

\section{Respiratorische Ursachen}

- Kinder mit „schwierigem Atemweg“ - problematische Intubation bei respiratorischer Insuffizienz oder Notfall-/Reanimationssituation,

- bekannte Obstruktionen der oberen Atemwege oder Fehlbildungen des Tracheobronchialbaums (oft syndromale Grunderkrankung mit kraniofazialer Anomalie oder Fehlentwicklung, z. B. Pierre-RobinSequenz etc.),

- neuromuskuläre Erkrankungen,

- akute Atemwegserkrankungen (Bronchitis, Pneumonie, Asthma etc.),

- pulmonale Rezirkulation mit vorbestehender erhöhter Atemarbeit,

- intrathorakale Störungen (Pleuraerguss, Pneumothorax etc.),

- Früh- und Neugeborene.

\section{Kardiozirkulatorische Ursachen}

- Systemkreislaufinsuffizienz jeglicher Genese, insbesondere schwere dilatative Kardiomyopathie oder akute Myokarditis,

- kreislaufrelevante Obstruktionen (z. B. kritische Aortenstenose, hypertroph-obstruktive Kardiomyopathie, hochgradige Pulmonalstenose, Aortenisthmusstenose etc.),

- pulmonale Rezirkulation mit resultierender erhöhter Atemarbeit (ShuntVitien),
- bekannte schwere pulmonale Hypertonie,

- kreislaufrelevante Arrhythmien,

- univentrikuläre Zirkulation,

- Früh- und Neugeborene,

- Art und Dauer des Eingriffs,

- Risiko des Eingriffs (mögliche Komplikationen).

\section{Kernaussage 3}

Kinderkardiologische Patienten mit kompensierter kardiopulmonaler Situation weisen für risikoarme und/oder kurze Prozeduren in moderater Sedierungstiefe kein erhöhtes Risikoprofil auf

\section{Kernaussage 4}

Ein erhöhtes Prozedurrisiko besteht bei

- akuter/chronischer kardiopulmonaler Beeinträchtigung

- komplexen/langwierigen Eingriffen

- tiefen Sedierungen/Allgemeinanästhesie

- Neugeborenen, Säuglingen und Kleinkindern

\section{Nüchternheitsgebot}

Für eine Intervention in Analgosedierung müssen alle Patienten zur Aspirationsprophylaxe nüchtern sein (d.h. feste Nahrung bis $6 \mathrm{~h}$, Milch bis $4 \mathrm{~h}$ und klare Flüssigkeit [kleine Mengen bis max. $200 \mathrm{ml}$ ] bis $2 \mathrm{~h}$ vor dem Eingriff).
Analgosedierungen werden in Ambulanzräumen, auf Normal-, Wach- und Intensivstationen sowie in speziellen Eingriffsräumen (CT, MRT, Herzkatheterlabor) durchgeführt (DGPK Umfrage 2018, [2]). Die Arbeitsplatzverhältnisse sollten entsprechend den prozedurbedingten Erfordernissen von Patient und Behandlungsteam angemessen groß und ausgestattet sein (Licht, Monitoring, Material, Kommunikationsmittel für Hilferufe), um eine rechtzeitige Erkennung von Problemen beim Patienten und dessen kardiorespiratorische Stabilisierung zu ermöglichen.

\section{Apparative und technische Voraussetzungen/Monitoring}

Die Risiken der Analgosedierung sind nach Art der Intervention und der Kompetenz des durchführenden Arztes unterschiedlich zu gewichten. Voraussetzung für die Patientensicherheit sind genügende Erfahrung und Routine des durchführenden Klinikers in der Analgosedierung, ein ausreichendes Monitoring sowie die verfügbare Infrastruktur (s. Abschn. „Räumlichkeit“). Insbesondere bei Patienten mit erhöhtem Prozedurrisiko müssen diese Voraussetzungen erfüllt sein (• Abb. 1; $[8,10])$.

In Abhängigkeit vom aktuellen klinischen Zustand des Patienten und seiner Grunderkrankung ist je nach Sedierungstiefe ein Monitoring notwendig, das individuell auf den Patienten abgestimmt ist. Nicht bei jedem Patienten und jeder Sedierungstiefe muss das komplette Monitoring eingesetzt werden. Anhaltspunkte für das notwendige Ausmaß des Monitorings liefert • Tab. 3.

Die Bewertung der hämodynamischen Beeinträchtigung ergibt sich aus der Grunderkrankung, dem Alter, der aktuellen Kreislaufsituation sowie dem geplanten Eingriff und seiner Dauer (-Abb. 1). Typische Beispiele hierzu sind in - Tab. 4 aufgeführt.

Zusätzlich sollten die Dauer und Komplexität der Eingriffe bewertet wer- 
Tab. 2 Klinische Effekte der Sedierungstiefen gemäß der Klassifikation der American Society of Anesthesiologists (ASA). (Modifiziert nach Zalunardo et al. [8])

\begin{tabular}{|c|c|c|c|c|c|c|c|}
\hline \multicolumn{2}{|c|}{ ASA-Stufe } & \multirow{2}{*}{$\begin{array}{l}\text { Bewusst- } \\
\text { sein } \\
\text { Wach }\end{array}$} & \multirow{2}{*}{$\begin{array}{l}\text { Reaktion auf Stimulation } \\
\text { Normale Reaktion auf Anspre- } \\
\text { chen }\end{array}$} & \multirow{2}{*}{$\begin{array}{l}\begin{array}{l}\text { Spontan- } \\
\text { atmung }\end{array} \\
\text { Nicht beein- } \\
\text { trächtigt }\end{array}$} & \multirow{2}{*}{$\begin{array}{l}\text { Schutzreflexe } \\
\text { Nicht beein- } \\
\text { trächtigt }\end{array}$} & \multirow{2}{*}{$\begin{array}{l}\text { Kreislauf } \\
\begin{array}{l}\text { Nicht beeinträch- } \\
\text { tigt }\end{array}\end{array}$} & \multirow{2}{*}{$\begin{array}{l}\text { Zusätzliches } \\
\text { Management } \\
\text { Überwiegend keines }\end{array}$} \\
\hline I & $\begin{array}{l}\text { Minimale } \\
\text { Sedierung }\end{array}$ & & & & & & \\
\hline II & $\begin{array}{l}\text { Moderate } \\
\text { Sedierung }\end{array}$ & $\begin{array}{l}\text { Schläfrig } \\
\text { (somnolent) }\end{array}$ & $\begin{array}{l}\text { Erweckbar; Wachphasen mit } \\
\text { normaler Reaktion auf Anspre- } \\
\text { chen und taktile Stimulation }\end{array}$ & $\begin{array}{l}\text { Ausreichend, } \\
\text { adäquat }\end{array}$ & $\begin{array}{l}\text { Nicht beein- } \\
\text { trächtigt }\end{array}$ & $\begin{array}{l}\text { In der Mehrzahl } \\
\text { der Fälle nicht } \\
\text { beeinträchtigt }\end{array}$ & Überwiegend keines \\
\hline III & $\begin{array}{l}\text { Tiefe Sedie- } \\
\text { rung }\end{array}$ & $\begin{array}{l}\text { Schlafend } \\
\text { (soporös) }\end{array}$ & $\begin{array}{l}\text { Nicht erweckbar, gezielte } \\
\text { Abwehrbewegungen auf } \\
\text { Schmerzreize }\end{array}$ & $\begin{array}{l}\text { Mit Beeinträch- } \\
\text { tigung ist zu } \\
\text { rechnen }\end{array}$ & $\begin{array}{l}\text { Mit Beeinträch- } \\
\text { tigung ist zu } \\
\text { rechnen }\end{array}$ & $\begin{array}{l}\text { In der Mehrzahl } \\
\text { der Fälle nicht } \\
\text { beeinträchtigt }\end{array}$ & $\begin{array}{l}\text { Sicherung der Atem- } \\
\text { wege/Atemhilfe kann } \\
\text { nötig sein }\end{array}$ \\
\hline IV & $\begin{array}{l}\text { Allgemein- } \\
\text { anästhesie }\end{array}$ & Bewusstlos & $\begin{array}{l}\text { Keine oder ungezielt; nicht } \\
\text { erweckbar }\end{array}$ & $\begin{array}{l}\text { Ggf. insuffizient } \\
\text { oder fehlend }\end{array}$ & $\begin{array}{l}\text { Ggf. aufgeho- } \\
\text { ben }\end{array}$ & Ggf. beeinträchtigt & $\begin{array}{l}\text { Sicherung der Atem- } \\
\text { wege/Beatmung } \\
\text { überwiegend not- } \\
\text { wendig }\end{array}$ \\
\hline
\end{tabular}

\begin{tabular}{|c|c|c|c|c|c|}
\hline & & \multicolumn{4}{|c|}{ Sedierungsstufe } \\
\hline \multicolumn{2}{|c|}{ Risikoprofil } & $\begin{array}{l}\text { I } \\
\text { Minimale } \\
\text { Sedierung }\end{array}$ & $\begin{array}{l}\text { II } \\
\text { Moderate } \\
\text { Sedierung }\end{array}$ & $\begin{array}{l}\text { III } \\
\text { Tiefe Sedie- } \\
\text { rung }\end{array}$ & $\begin{array}{l}\text { IV } \\
\text { Allgemein- } \\
\text { anästhesie }\end{array}$ \\
\hline A & $\begin{array}{l}\text { Herzfehler/-erkrankung ohne } \\
\text { hämodynamische Beeinträch- } \\
\text { tigung } \\
\text { Rein diagnostisch } \\
\text { Kurze Dauer }\end{array}$ & Klinisch & Basis & Erweitert & Komplett \\
\hline B & $\begin{array}{l}\text { Geringe hämodynamische } \\
\text { Beeinträchtigung } \\
\text { Diagnostik bzw. einfacher } \\
\text { Eingriff } \\
\text { Kurze Dauer }\end{array}$ & Klinisch & Basis & Erweitert & Komplett \\
\hline C & $\begin{array}{l}\text { Mittelschwere hämodynami- } \\
\text { sche Beeinträchtigung } \\
\text { Mittleres Risiko } \\
\text { (Evtl.) längere Prozedur }\end{array}$ & $\begin{array}{l}\text { Basis/ } \\
\text { Erweitert }\end{array}$ & Erweitert & Komplett & Komplett \\
\hline D & $\begin{array}{l}\text { Schwere hämodynamische } \\
\text { Beeinträchtigung } \\
\text { Risikoeingriff } \\
\text { Lange Prozedur }\end{array}$ & Komplett & Komplett & Komplett & Komplett \\
\hline \multicolumn{6}{|c|}{$\begin{array}{l}\text { A, B, C, D Beispiele der Risikoprofile: - Tab. } 4 \\
\text { Klinisches Monitoring klinische Überwachung anhand Atemtiefe, Atemfrequenz, Hautfarbe und } \\
\text { Puls } \\
\text { Basis-Monitoring klinische Überwachung plus Pulsoxymetrie, ggf. nichtinvasive Blutdruckmessung } \\
\text { Erweitertes Monitoring Basis-Monitoring, einschließlich nichtinvasiver Blutdruckmessung plus } \\
\text { Elektrokardiogramm (EKG), i.v.-Zugang } \\
\text { Komplettes Monitoring erweitertes Monitoring plus ggf. zusätzliche apparative Überwachung, z. B. } \\
\text { Kapnometrie, Nahinfrarotspektroskopie (NIRS) }\end{array}$} \\
\hline
\end{tabular}

den: Eine kurze, nichtinvasive diagnostische Untersuchung (z.B. Echokardiographie) bedarf nur eines Monitorings mit geringem Aufwand (klinisch/Basis); ein invasiver Eingriff mit höherem Risiko (z.B. Perikarddrainage) dagegen zumindest eines erweiterten Monitorings. Länger dauernde Eingriffe (MRT beim Vorschulkind, elektrophysiologi- sche Untersuchung) sollten mit einem kompletten Monitoring erfolgen, auch wenn nur ein geringes Risiko besteht.

Alle im Rahmen der Patientenüberwachung eingesetzten Geräte müssen regelmäßig gewartet und auf ihre Funktionsfähigkeit geprüft werden. Die Medikamente müssen regelmäßig auf Vollständigkeit und Verfallsdatum geprüft werden. Eine Dokumentation der Prüfverfahren ist obligat.

\section{Personal und Zuständigkeit}

Nicht immer ist der Arzt, der den Eingriff durchführt, während der diagnostischen oder therapeutischen Maßnahme in der Lage, die Vitalfunktionen des Patienten in ausreichendem Maß zu überwachen und ggf. auf Veränderungen zu reagieren. Daher ist bei vielen Analgosedierungen eine weitere, entsprechend qualifizierte und nicht in die diagnostische oder therapeutische Maßnahme involvierte Person erforderlich, deren wesentliche Aufgaben die Durchführung und die Überwachung des Analgosedierungsverfahrens sind. Analgosedierungen der Stufen I und II bzw. Analgosedierungen bei kurzen Prozeduren erfolgen durch hierfür qualifiziertes nichtärztliches Personal. Hierbei trägt der den Eingriff durchführende Arzt die Verantwortung auch für die Analgosedierung.

Tiefe Analgosedierungen ( $\geq$ Stufe III) erfordern für die Durchführung und Überwachung weitere, entsprechend qualifizierte personelle Unterstützung. Auf das bereits in der Kardiologie als effektiv und sicher etablierte sowie in den meisten kinderkardiologischen Zentren in der klinischen Praxis genutzte Konzept der "nurse-administered propofol sedation" wird ausdrücklich hingewiesen (NAPS, $[3,6]$ ). Wird ein zweiter Arzt hinzugezogen, muss die Verantwortlichkeit für die Analgosedierung geregelt sein. 
Tab. 4 Beispiele hämodynamischer Beeinträchtigung

A Herzfehler/-erkrankung ASD II, kleiner VSD, milde PS, AS etc.

ohne hämodynamische

Beeinträchtigung

Älteres Kind, Jugendlicher (ZVK > 10 J; MRT > 10 J)

Kleiner/kürzerer Eingriff (Pleurapunktion)

B Geringe

hämodynamische

Beeinträchtigung

C Mittelschwere

hämodynamische

Beeinträchtigung

Neugeborenes mit AVSD ohne Medikamente

Fontan-Palliation ohne aktuelle klinische Beeinträchtigung

Diagnostischer HK bei klinisch unbeeinträchtigtem Patienten

Myokarditis mit Arrhythmie, aber erhaltener systolischer Ventrikelfunktion

Neugeborenes mit Tachydyspnoe

Prozedur $>30$ min bei Säuglingen mit Shunt-Vitium

D Schwere

hämodynamische

Beeinträchtigung

Kardiomyopathie mit reduzierter systolischer Ventrikelfunktion

Pulmonalarterielle Hypertonie mit systemischem oder supra-

systemischem Druck im rechten Ventrikel

AS Aortenstenose, ASD Atriumseptumdefekt, AVSD atrioventrikulärer Septumdefekt, $H K$ Herzkatheter, J Jahre, MRT Magnetresonanztomographie, PS Pulmonalstenose, VSD Ventrikelseptumdefekt, ZVK zentraler Venenkatheter

\section{Kernaussage 5}

- Eine Analgosedierung kann unter ärztlicher Verantwortung an geschultes nichtärztliches Personal delegiert werden

- Bei Sedierungen der Stufen III und IV muss eine weitere Person mit ausreichend Erfahrung in Theorie und Praxis der Analgosedierung hinzugezogen werden, die nicht in den Eingriff involviert ist

\section{Qualifikationen}

Ärztliches Personal. Moderate Analgosedierungen (Stufen I und II) können von Ärzten aller Fachrichtungen ohne spezielle Zusatzweiterbildung durchgeführt werden, wenn das Verfahren bzw. der Eingriff zu keiner Beeinträchtigung der Schutzreflexe oder der Vitalfunktionen führt.

Bei höheren Sedierungsgraden kann es aufgrund der fließenden und teilweise unvorhersehbaren Übergänge in tiefere Sedierungsstadien zum Verlust der Ansprechbarkeit bzw. zur Beeinträchtigung der Vitalfunktionen und Schutzreflexe kommen. Daher müssen diese tiefen Sedierungen von ärztlichem und nichtärztlichem Personal mit entsprechender Qualifikation und Erfahrung durchgeführt werden, die in der Lage sind, bedrohliche Verläufe zu erkennen und entsprechend zu behandeln.
Die Fachrichtung des Arztes hat nach wissenschaftlicher Datenlage keinen Einfluss auf die Komplikationsrate [11].

Prinzipiell ist jede/jeder approbierte Ärztin/Arzt einschließlich Arzt/Ärztin in Weiterbildung zum/zur Facharzt/ Fachärztin für Kinderheilkunde und Jugendmedizin bzw. Kinderkardiologie oder Intensivmedizin aufgrund der Weiterbildungscurricula geeignet, mit entsprechender Erfahrung eine Analgosedierung durchzuführen (Musterweiterbildungsordnung BÄK [12]). In der Praxis der deutschsprachigen Kinderheilkunde und Jugendmedizin werden Sedierungsmaßnahmen überwiegend von Pädiatern durchgeführt [10]. Die aktuelle Umfrage der DGPK 2018 bestätigt dieses Vorgehen auch für kinderkardiologische Patienten [2]. Gründe für die Durchführung von Analgosedierungen durch Anästhesisten beruhen im Wesentlichen auf lokalen Organisationsstrukturen.

Nichtärztliches Personal. Analgosedierungen können von Ärzten aller Fachrichtungen an nichtärztliches Personal mit entsprechenden Kenntnissen und Erfahrungen delegiert werden.

Bei der Delegation der Analgosedierung an nichtärztliches Personal muss dieses eine entsprechende Unterrichtung (Schulung) in Theorie und Praxis der Analgosedierung erhalten. Dies beinhaltet auch das Erkennen von Komplikationen, die Besonderheiten der eingesetzten $\mathrm{Me}$ - dikamente, Basiswissen im Atemwegsmanagement (z. B. Beutelbeatmung) sowie Kenntnisse und Fähigkeiten der kardiopulmonalen Reanimation.

Bei Gesundheitspflegern und -pflegerinnen mit Fachweiterbildung Intensivpflege oder Anästhesie oder auch langjährig erfahrenen Pflegern/Pflegerinnen im Bereich Kinderkardiologie bzw. Intensivmedizin kann von diesen erforderlichen Kenntnissen und Erfahrungen ausgegangen werden. Abteilungsinterne Curricula und „standard operating procedure" (SOP) mit entsprechender Dokumentation werden in diesem Zusammenhang empfohlen $[10,13,14]$.

\section{Prozedurale Durchführung - Ablauf und Überwachung der Analgosedierung}

Die zugrunde liegende kardiale Erkrankung und der klinische Status des Patienten, einschließlich seiner aktuellen hämodynamischen Situation, müssen dem Team, das den Eingriff und die dazu notwendige Analgosedierung vornimmt, bekannt sein. Der Arzt, der den Eingriff durchführt, übernimmt die Verantwortung für den korrekten Ablauf der gesamten Prozedur. Dies schließt die Analgosedierung ein, wenn für diese keine zusätzliche Ärztin/kein zusätzlicher Arzt vorgehalten wird. In Abhängigkeit von der zu erwartenden Schwere des Eingriffs und dem Risikoprofil des Patienten kann die Anwesenheit eines zweiten ausschließlich für die Sedierung zuständigen Arztes notwendig werden. Wenn die Analgosedierung von ärztlichem Personal durchgeführt wird, müssen Verantwortung und Zuständigkeit geregelt sein.

Die Analgosedierung muss von einem Team, bestehend aus adäquat geschultem ärztlichem und ggf. nichtärztlichem Personal durchgeführt werden. Alle an der Sedierung beteiligten Personen müssen spezielle Kenntnisse in Theorie und Praxis des Komplikationsmanagements von akuten respiratorischen und kreislaufrelevanten Komplikationen aufweisen. Es ist erforderlich, während des Eingriffs eine durchgehende Überwachung der Vitalparameter (Untersuchungs-, Monitoring-Raum) zu gewährleisten. Die Atemwegssicherung und Wiederherstellung der übrigen Vitalfunktionen müssen 
jederzeit gewährleistet sein. Mindestens ein Mitglied des Behandlungsteams muss in der Lage sein, Beatmung über Maske und Beutel sicher zu beherrschen sowie bei Bedarf eine suffiziente Sicherung des Atemwegs vorzunehmen.

Die Analgosedierung, einschließlich deren Überwachung und ggf. die Wiederherstellung vitaler Funktionen beim $\mathrm{Pa}$ tienten erfolgen unter ärztlicher Verantwortung und während des Eingriffs durch eine vom Eingriff unabhängige Person. Eine qualifizierte Fachperson muss für die Verabreichung der Sedativa und Analgetika während der Intervention sowie die Überwachung des Patienten vor, während und nach der Intervention zur Verfügung stehen.

Der für die Analgosedierung verantwortliche Arzt muss Erfahrung in der Behandlung von Notfällen sowie in der pädiatrischen Intensiv- und Notfallmedizin haben. Zur Erlangung dieser Fähigkeit sind z. B. 6 Monate Weiterbildung auf einer pädiatrischen, neonatologischen oder interdisziplinären Intensivstation oder kinderkardiologischen „Intermediate-care"(IMC)-Station mit Kenntnissen im Bereich Sedierung (Atemwegsmanagement, Pharmakotherapie etc.) sowie Notfallsituationen (Reanimation) erforderlich. Dem Themenbereich Analgosedierung muss in ausreichendem $\mathrm{Ma} ß$ in der Aus- und Weiterbildung Raum eingeräumt werden, damit das Notfallmanagement und die Kenntnisse der in der jeweiligen Klinik verwendeten Analgetika und Sedativa vermittelt und erlernt werden können.

\section{Kernaussage 6}

Die kardiale Grunderkrankung, die aktuelle hämodynamische Situation und der funktionelle Status des Patienten müssen dem Team, das den Eingriff und die Analgosedierung vornimmt, bekannt sein.

Der die Intervention durchführende Arzt darf die Analgosedierung während der Intervention nicht allein durchführen, kann und soll aber entsprechend qualifiziertes Fachpersonal anweisen. Vor und nach der Intervention kann der durchführende Arzt die Analgo- sedierung beginnen bzw. fortführen. Im Notfall oder bei Beeinträchtigungen von Atmung und/oder Kreislauffunktion muss ein zweiter Arzt umgehend verfügbar sein, der die Steuerung der Analgosedierung übernehmen kann. Eine zusätzliche mit dem Prozedere und den Örtlichkeiten vertraute Person muss unmittelbar abruf- und verfügbar sein, um bei einer kardiopulmonalen Beeinträchtigung des Patienten assistieren zu können.

Nach Beendigung des Eingriffs und der dafür notwendigen Analgosedierung ist eine weitere Überwachung des Patienten bis zum Aufwachen mit vollständiger Rückkehr der Schutzreflexe bei suffizienter Spontanatmung - mindestens aber für $60 \mathrm{~min}$ (bei Verwendung von Opioiden mindestens $4 \mathrm{~h}$ ) - durch entsprechend geschultes und qualifiziertes Personal notwendig (z.B. IMC-Station). Hierbei ist neben der obligaten klinischen (Vigilanz, Atemexkursionen des Thorax, Atemgeräusche, Farbe der Lippen) auch eine apparative Überwachung (Pulsoxymetrie, EKG, Blutdruck) notwendig. Dies gilt ebenso für den Transport nach dem Eingriff zum Ort der weiteren Überwachung. Es darf keine Überwachungslücke zwischen dem Ort der Maßnahme und dem Erholungsraum geben.

\section{Prozessqualität}

- Die Aufklärung über die zur Durchführung einer diagnostischen oder therapeutischen Maßnahme notwendigen Analgosedierung muss erfolgen und schriftlich oder in elektronischer Form dokumentiert werden. Dieses geschieht im Rahmen der Aufklärung für die geplante Prozedur bzw. ist Teil der geplanten Prozedur und benötigt keine gesonderte Aufklärung. Gesonderte Vordrucke zur Aufklärung über Sedierung sind hilfreich, aber nicht obligat.

- Die Aufklärung über die Sedierung sollte neben dem generellen Ablauf sowie Wirkungen der sedierenden und analgetischen Medikamente auch mögliche Alternativen, Konsequenzen und Komplikationen beinhalten.
- Innerhalb einer Institution sollen nur allen Beteiligten vertraute Verfahren und bekannte Medikamente zur Analgosedierung angewendet werden.

- Eine Analgosedierung soll unter Zielfestlegung der Sedierungsstufe mit dem Ziel der Toleranz der Prozedur erfolgen.

- Ein Notfallalgorithmus („Rettungskette") muss vorhanden und bekannt sein.

- Verabreichte Medikamente (Zeit und Dosis), Beginn und Ende der Maßnahmen sowie die bei tiefer Sedierung (Stufe III) gemessenen Vitalparameter müssen regelmäßig (z.B. im 5- bis 10-min-Intervall) dokumentiert werden.

- Eine Möglichkeit der Überwachung nach der Analgosedierung, einschließlich Schmerzbehandlung, muss vorhanden sein.

- Entlassungskriterien bei ambulanten Patienten sind definiert.

\section{Medikamente}

- Die Wahl der Medikamente steht in der Kompetenz und Verantwortung des behandelnden Arztes bzw. wird in Verfahrensanweisungen (SOP) geregelt. Auf eine detaillierte Auflistung der verwendeten Medikamente oder Dosierungen wird im Rahmen dieses Positionspapieres bewusst verzichtet.

- Als Sedativa kommen abhängig von Zentrum und Indikation unterschiedliche Medikamente zum Einsatz [2]. Es sollen möglichst kurz wirksame und gut steuerbare Sedativa und Analgetika eingesetzt werden.

- Für einen Eingriff mit höherer Sedierungsstufe erfolgen $[2,15,16]$ :

- eine Prämedikation (mit z.B. Benzodiazepinen, Midazolam),

- eine Sedierung (mit z. B. Propofol),

- eine Analgesie (mit z.B. Ketamin oder kurz wirksamen Opioiden),

- eine entsprechende Lokalanästhesie als Alternative oder als Ergänzung zur systemischen Analgesie,

- ggf. eine periinterventionelle Analgesie (mit z. B. Ibuprofen, Metamizol). 


\section{Überwachung nach der Intervention und Entlassungs- kriterien}

- Die Erholungsphase des Patienten muss in einer geeigneten Räumlichkeit mit personeller Überwachung erfolgen.

- Bei höherer Sedierungsstufe ( $\geq$ Stufe III) muss eine Monitor-Überwachung (EKG, Pulsoxymetrie, ggf. nichtinvasive Blutdruckmessung) durchgeführt werden.

- Eine Entlassung ist nur bei stabilen Vitalparametern und subjektivem Wohlbefinden des Patienten möglich.

- Der behandelnde Arzt entscheidet nach entsprechender klinischer Untersuchung über den Entlassungszeitpunkt.

\section{Ausbildung/interne Qualitäts- kontrolle}

- Ein allgemeingültiges Ausbildungscurriculum für die Durchführung von Sedierungen bei kinderkardiologischen Patienten existiert bisher nicht. Abteilungsinterne Curricula und SOP mit entsprechender Dokumentation werden in diesem Zusammenhang empfohlen.

- Ärzte und Assistenzpersonal sind regelmäßig und periodisch in den Maßnahmen der Analgosedierung, Sicherung der Atemwege und Reanimation zu trainieren.

- Besprechungen im Team über die Sedierung, einschließlich regelmäßiger Morbiditäts- und Mortalitätsbesprechungen, sollten durchgeführt werden, um in regelmäßigen $\mathrm{Ab}$ ständen die Prozesse zu optimieren sowie die Qualität der Sedierung zu hinterfragen und zu optimieren $[10$, $13,14]$.

\section{Korrespondenzadresse}

Univ. Prof. Dr. Nikolaus Haas

Abteilung Kinderkardiologie und Pädiatrische Intensivmedizin, Klinikum der LudwigMaximilians-Universität, Campus Großhadern Marchioninistr. 15, 81377 München, Deutschland

Nikolaus.Haas@med.uni-muenchen.de

\section{Einhaltung ethischer Richtlinien}

Interessenkonflikt. H. Bertram, H. Sauer und N. Haas geben an, dass für diesen Artikel kein Interessenkonflikt besteht.

Für diesen Beitrag wurden von den Autoren keine Studien an Menschen oder Tieren durchgeführt. Für die aufgeführten Studien gelten die jeweils dort angegebenen ethischen Richtlinien.

\section{Literatur}

1. Neuhäuser C, Wagner B, Heckmann M, Wigand MA, Zimmer KP (2010) Analgosedierung für schmerzhafte Eingriffe bei Kindern und Jugnedlichen. Dtsch Ärztebl Int 107(14):241-247

2. Sauer H, Bertram H, Dietl M et al (2019) Analgosedierung bei kinderkardiologischen Patienten. Ergebnisse einer deutschlandweiten Umfrage. Monatsschr Kinderheilkd. https://doi. org/10.1007/s00112-019-0744-y

3. Tilz RR, Chun KRJ, Deneke T, Kelm M, Piorkowski C, Sommer P, Stellbrink C, Steven D (2017) Positionspapier der Deutschen Gesellschaft für Kardiologie zur Kardioanalgosedierung. Kardiologe 11:369-382

4. Hanslik A, Moysich A, Laser KT, Mlczoch E, Kececioglu D, Haas NA (2014) Percutaneous closure of atrial septal defects in spontaneously breathing children under deep sedation: a feasible and safe concept. Pediatr Cardiol 35(2):215-222

5. Pees $C$, Haas NA, Ewert $P$, Berger F, Lange PE (2003) Comparison of analgesic/sedative effect of racemic ketamine and $\mathrm{S}(+)$-ketamine during cardiac catheterization in newborns and children Pediatr Cardiol 24(5):424-429

6. Lamond DW (2010) Review article: Safety profile of propofol for paediatric procedural sedation in the emergency department. Emerg Med Australas 22:265-286

7. ASA (2004) Continuum of depth of sedation: Definition of general anesthesia and levels of sedation/analgesia. Committee of Origin: Quality Management and Departmental Administration (Approved by the ASA House of Delegates on October 27, 2004, and amended on October 21, 2009)

8. Zalunardo M, Krayer S, Brunner T, Walder B, Bauerfeind $\mathrm{P}$, Hartmeier S, Ammann P, Weilenmann D Jacob AL, Franzen D (2016) Empfehlungen und Standards für die Analgosedierung durch NichtAnästhesisten. Konsensuspapier der Schweizer Gesellschaft für Anästhesiologie und Reanimation. Swiss Med Forum 16(45):969-972 (Update 2018)

9. ASA (2014) Physical status classification system; last approved by the ASA House of Delegates on October 15, 2014; Homepage der Amer- ican Society of Anesthesiology. www.asahq. org/resources/clinical-information/asa-physicalstatus-classification-system. Zugegriffen am 24. Januar 2019

10. Sauer H, Lobenhofer M, Meyer S (2018) Analgosedierung für diagnostische und interventionelle Prozeduren in der Pädiatrie. DIVI 9(2):48-54

11. Couloures KG, Beach M, Cravero JP, Monroe KK Hertzog JH (2011) Impact of provider specialty on pediatric procedural sedation complication rates. Pediatrics 127:e1154-e1160

12. Bundesärztekammer (Arbeitsgemeinschaft der deutschen Ärztekammern) (2015) (Muster-)Weiterbildungsordnung 2003 in der Fassung vom 23.10.2015. www.bundesaerztekammer.de/ fileadmin/user_upload/downloads/pdf-Ordner/ Weiterbildung/MWBO.pdf. Zugegriffen am 24. Januar 2019

13. Sauer H, Grünzinger L, Pfeifer J, Lieser U, AbdulKhaliq H (2016) Sedation and analgosedation performed by pediatricians-Experience made with the implementation of an in-house sedation standard. Wien Med Wochenschr 166:54-61

14. Sauer H, Haase R, Lieser U, Horneff G (2008) Vorbereitung und Monitoring im Rahmen von Sedierung und Analgosedierung durch Fachärzte und Weiterbildungsassistenten in der Kinder- und Jugendmedizin. Klin Pädiatr 220:189-195

15. Philippi-Höhne C, Becke K, Wulff B, Schmitz B, Strauß J, Reinhold P (2010) Analgosedierung für diagnostische und therapeutische Maßnahmen im Kindesalter. Anästh Intensivmed 51:S603-S614

16. American Academy of Pediatrics, American Academy of Pediatric Dentistry, Coté CJ, Wilson S, Work Group on Sedation (2006) Guidelines for monitoring and management of pediatric patients during and after sedation for diagnostic and therapeutic procedures: An update. Pediatrics 118:2587-2602 


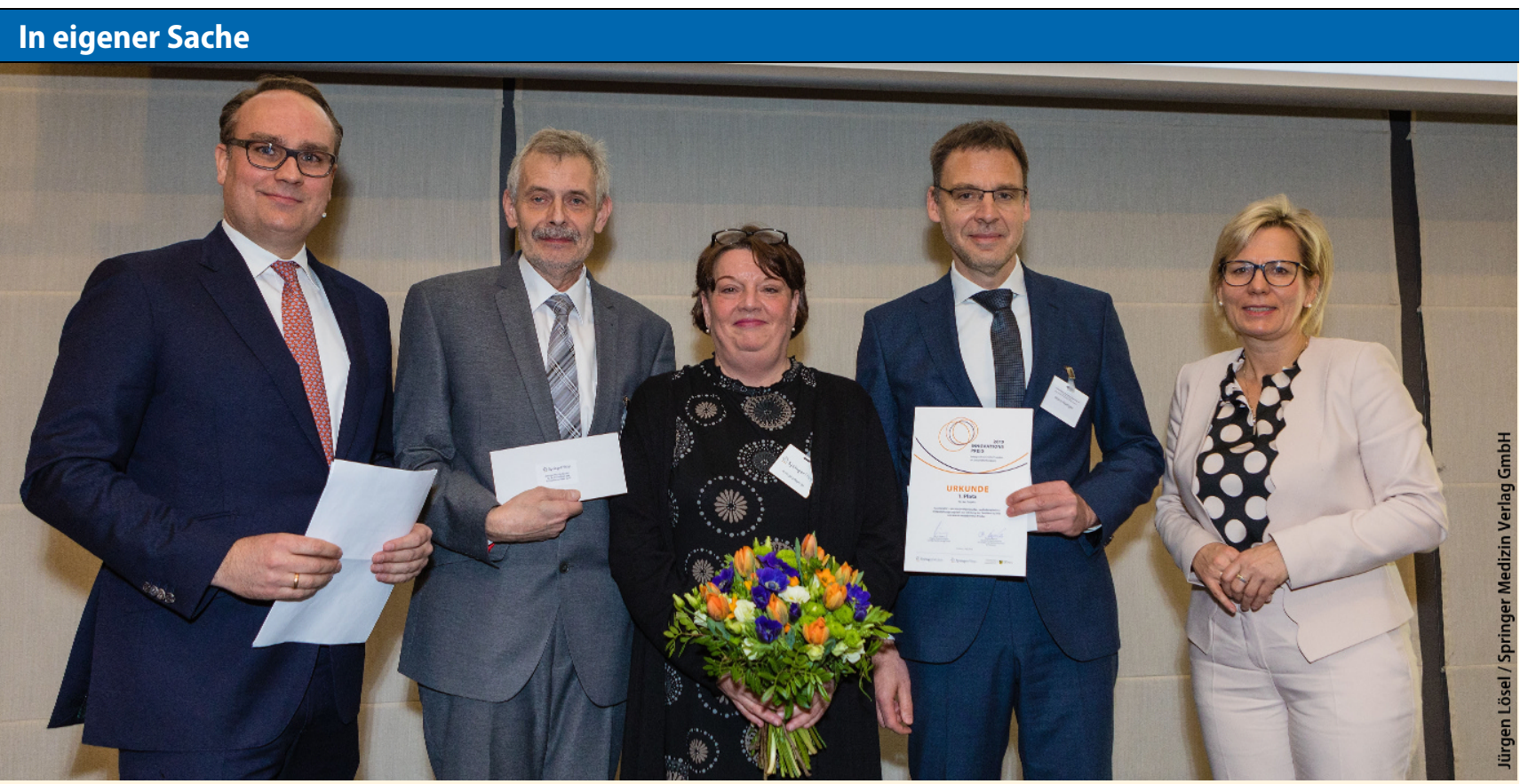

\section{Versorgung von Frühchen: „Eltern, hört meine Signale!“}

Siegerprojekt des Innovationspreises 2019 für interprofessionelle Projekte im Gesundheitswesen

\section{Am Uniklinikum Dresden werden Eltern von frühgeborenen Kindern durch ein ausgezeichnetes Versorgungsprojekt unterstützt. Eines der Ziele: Von Beginn an Bindung zum Frühchen aufzubauen.}

Die medizinische Versorgung Frühgeborener ist eine Domäne der Hochleistungsmedizin. Manchmal drohen die Eltern dabei aus dem Blick zu geraten. „Die Eltern Frühgeborener sind oft extrem belastet", berichtet Professor Mario Rüdiger, Leiter Fachbereich Neonatologie und Pädiatrische Intensivmedizin am Universitätsklinikum Dresden. „Die Erkrankung ihres Kindes und der mitunter Monate dauernde Krankenhausaufenthalt machen es innen schwer, die Bindung zu ihrem Kind aufzubauen - mit negativen Folgen für sich und ihr Kind."

Rüdiger und sein Team haben im Laufe von zehn Jahren ein komplexes Versorgungsangebot für Eltern entwickelt, etabliert und evaluiert. „Wir stärken damit die intuitive elterliche Kompetenz, die bei Eltern Frühgeborener oft nicht gleich voll verfügbar ist." Das Projekt mit dem Namen „FamilieNETZ - ein interprofessionelles, multidisziplinäres Unterstützungsangebot zur Stärkung der Familien zu früh und krank neugeborener Kinder" hat beim von Springer Medizin und Springer Pflege verliehenen Innovationspreis 2019 für interprofessionelle Projekte im Gesundheits- wesen den ersten Platz errungen.

Grundlage des Angebots sind unter anderem evaluierte Schulungsprogramme, die aufeinander abgestimmt und bedarfsgerecht kombiniert werden. So können Eltern etwa im Programm „Frühe Signale“ lernen, prototypische Signale von Frühchen zu verstehen. „Denn sie senden andere Signale aus als reife Neugeborene", so Rüdiger. Die Eltern werden angeleitet, ihr Kind - noch in der Klinik - selbst zu baden und diese Zeit für abgestimmte Interaktionen zu nutzen. In einem Videofeedback dazu reflektieren sie das sich entwickelnde Eltern-Kind-Team.

Eine Studie zeigte: „Wenn Eltern geschult sind, schauen ihre Kinder sie beim Baden oft und lange an, weil sie prompt und adäquat auf ihr Kind reagieren", erklärt PD Dr. Jörg Reichert, Psychologe und Leiter des FamilieNETZ. „Das zeugt von den größer werdenden Aufmerksamkeitsspannen und von sicherer Bindung." Weitere Erfolge solcher Interventionen sind durch internationale Studien belegt: Verminderung von Stress und Angst bei den Eltern, dafür erleben sie mehr Selbstwirksamkeit. Bei den Kindern wird Hirnreifung und neurologische Entwicklung positiv beeinflusst.

Außer Vorteilen für Eltern und Kind ist auch ein ökonomischer Effekt zu verzeichnen: „Wir konnten für die bei uns betreuten Kinder zeigen, dass die Krankenkasse bei einem Einsatz von 200000 Euro im Jahr während der ersten zwei Lebensjahre etwa 400000 Euro Gesundheitskosten einspart - vor allem durch weniger und kürzere Krankenhausaufenthalte", sagt Rüdiger. Er und sein Team setzen sich für einen bundesweiten Transfer des Angebots in die Regelversorgung ein: Inzwischen sind eine AWMF-Leitlinie, eine Weiterbildung sowie eine OPS-Ziffer entstanden. „Wir hoffen, dass die OPS-Ziffer bald mit einem festen Geldwert hinterlegt ist, sodass die Leistungen auch abrechenbar sind." Es gäbe eine Nachfrage anderer Perinatalzentren, so Rüdiger. „Schließlich wird die Nachhaltigkeit der aufwändigen Erstversorgung von Frühgeborenen mit dem Unterstützungsangebot für Eltern deutlich gestärkt."

Bild v.l.n.r: Falk Miekley, Director Professional Care, Springer Medizin Verlag GmbH; PD Dr. Jörg Reichert, Projektverantwortlicher, Leiter des psychologisch-sozialmedizinischen Versorgungsbereiches, Universitätsklinikum Dresden; Marion Sommerfeld, Projektverantwortliche, Pflegerische Leitung FamilieNETZ , Universitätsklinikum Dresden; Prof. Dr. Mario Rüdiger, Projektverantwortlicher, Leiter des Fachbereiches Neonatologie und Pädiatrische Intensivmedizin, Universitätsklinikum Dresden; Barbara Klepsch, Sächsische Staatsministerin für Soziales und Verbraucherschutz

Quelle: Ärztezeitung/A. Nolte 